- ACORN Australan colege of | JOURNAL OF PERIOPERATIVE NURSING

\title{
Variations in COVID-19 airway management and preparedness among Victorian hospitals
}

Follow this and additional works at: https://www.journal.acorn.org.au/jpn

Part of the Anesthesiology Commons, Critical Care Commons, and the Infectious Disease Commons (c) (i)

This work is licensed under a Creative Commons Attribution 4.0 License.

\section{Recommended Citation}

Jordan, Kaylee A.; Sindoni, Teresa M.; Segal, Reny; Lee, Keat; Krieser, Roni B.; Mezzavia, Paul; Chen, Yinwei; and $\mathrm{Ng}$, Irene (2021) "Variations in COVID-19 airway management and preparedness among Victorian hospitals," Journal of Perioperative Nursing: Vol. 34 : Iss. 4 , Article 3.

Available at: https://doi.org/10.26550/2209-1092.1142

https://www.journal.acorn.org.au/jpn/vol34/iss4/3

This Article is brought to you for free and open access by Journal of Perioperative Nursing. It has been accepted for inclusion in Journal of Perioperative Nursing by an authorized editor of Journal of Perioperative Nursing. 


\section{Variations in COVID-19 airway management and preparedness among Victorian hospitals}

\section{Cover Page Footnote}

The authors acknowledge and thank the anaesthetic departments that participated in this audit, and their representative anaesthetists who assisted with the provision of information for its completion. 


\section{Authors}

Dr Kaylee A Jordan

MBBS (Hons), DRANZCOG, FANZCA

Staff Consultant Anaesthetist, Royal

Melbourne Hospital, Parkville, Australia

Ms Teresa Sindoni

BNurs, GradCert

(ClinNurs) CNS, Anaesthetic \& Recovery,

Royal Melbourne Hospital Research

nurse, Royal Melbourne Hospital, Parkville,

Australia

Professor Reny Segal

MBChB, FANZCA

Staff Consultant Anaesthetist, Royal

Melbourne Hospital, Parkville, Australia

Clinical Professor, University of Melbourne,

Parkville, Australia

Dr Keat Lee

MBBS, PDip (ClinUS), FANZCA

Head of Clinical Operations, Royal

Melbourne Hospital, Parkville, Australia

Honorary Clinical Senior Lecturer,

University of Melbourne, Parkville, Victoria, 3050, Australia.

Dr Roni B Krieser

MClinUS, MBBS(Hons),

GradDip (PeriopEcho), FANZCA Staff

Consultant Anaesthetist, Royal Melbourne

Hospital, Parkville, Australia

Dr Paul M Mezzavia

MClinUS, MBBS,

FANZCA Staff Consultant Anaesthetist,

Royal Melbourne Hospital, Parkville,

Australia

Ms Yinwei Chen

BNurs, GradCert

(ClinNurs) CNS, Anaesthetic \& Recovery,

Royal Melbourne Hospital Research

nurse, Royal Melbourne Hospital, Parkville, Australia

Dr Irene Ng

MClinRes, MBBS, GCert

(CritCareEcho), FANZCA Staff Consultant

Anaesthetist, Royal Melbourne Hospital,

Parkville, Australia Honorary Fellow,

University of Melbourne, Parkville,

Australia

\section{Corresponding author}

Dr Kaylee A Jordan

MBBS (Hons), DRANZCOG, FANZCA

Staff Consultant Anaesthetist, Royal

Melbourne Hospital, Parkville, Australia

kaylee.jordan@mh.org.au

\section{Variations in COVID-19 airway management and preparedness among Victorian hospitals}

\section{Summary}

The COVID-19 pandemic presents significant concerns surrounding the risk of transmission to health care workers involved in airway management of patients with suspected or known infection. Limited evidence has been available to guide the preparation of staff, intubation environments, team structure and personal protective equipment. Our study invited Victorian hospitals to complete a survey on their airway management practices and protocols, in order to assess the degree of variability in practice and preparedness. Twenty hospitals responded in September 2020, during Victoria's second wave of COVID-19. Forty percent had dedicated COVID-19 intubation teams, all including consultant anaesthetists. Seventy-five percent had negatively pressured dedicated intubation rooms. All provided airborne precautions including N95 masks for airway and cardiac arrest management of suspected or confirmed COVID-19 positive patients, with 35 per cent providing N95 mask fit testing and 15 per cent providing powered air purifying respirators or elastomeric respirators. Thirty-five percent provided airborne precautions for cardiac arrest management of patients not suspected to be COVID-19 positive. Significant inter-hospital variations were reported in airway management practices, such as preoxygenation, bag-mask ventilation, medications and techniques to minimise aerosolisation. Although some of this variation was likely due to individual hospital infrastructure and resource limitations, it would be ideal to achieve a more consistent, standardised approach across Victorian hospitals. This study may highlight areas for improvement for some hospitals. These areas for improvement may include consideration of the establishment of COVID-19 intubation teams in at least major metropolitan hospitals, N95 mask fit testing and the use of airborne precautions for cardiac arrest management during times of increased community prevalence of COVID-19.

\section{Introduction}

The World Health Organization (WHO) declared the 2019 novel coronavirus (COVID-19) to be a pandemic on 11 March 20201. Despite recent experiences with disease outbreaks, such as influenza, EBOLA and Middle East Respiratory Syndrome Coronavirus (MERS-CoV), the combination of high transmission and mortality rates of COVID-19 made this pandemic an unprecedented event, for which most health care systems in the world were not adequately prepared ${ }^{1,2}$

Health care workers are at increased risk of developing the infection, due to their close contact with patients and each other, and their involvement in procedures that may increase the dispersion of contaminated fluid or aerosols $\mathrm{s}^{3,4}$. One study showed that one in ten health care workers who were involved in tracheal intubation of patients with suspected or confirmed COVID-19 subsequently reported a COVID-19 
outcome, defined as either being diagnosed with new COVID-19 infection or requiring self-isolation or hospitalisation with new symptoms 5 . Hospitals around the world, including those in Australia, had to promptly develop airway management guidelines and protocols with very limited supporting literature at the beginning of the pandemic ${ }^{6}$. Several guidelines then gradually became available as new information emerged, to guide medical professionals on airway management of suspected and confirmed COVID-19 positive patients, including those released by the Safe Airway Society? the Anesthesia Patient Safety Foundation ${ }^{8}$, the Australian Society of Anaesthetists ${ }^{3}$ and the Australian and New Zealand Intensive Care Society ${ }^{9}$. However, variations in practice among hospitals have been reported ${ }^{10}$.

Recent concerns have been raised about particular features of Victoria's health system, and whether this may play a role in the significantly higher incidence of COVID-19 that has been observed in comparison to other states ${ }^{11}$. Most of the states in Australia have overarching health structures and central sources of guidelines and standards. In contrast, in Victoria there are six Primary Health Networks (PHNs) and 88 Local Health Care Authorities ${ }^{12}$. Each of the PHNs operates as its own separate entity, making it challenging to integrate and share information and resources across different networks. The aim of this study was to examine how different hospitals in Victoria prepare, coordinate and conduct the airway management of patients with suspected or confirmed COVID-19. This information can be used as a reference for hospital staff who are involved in planning COVID-19 airway management.

\section{Methods}

After obtaining approval from Melbourne Health Human Research Ethics Committee (QA2020128), we invited the hospitals that are affiliated with the University of Melbourne to participate in this study ${ }^{13}$. We included all the adult hospitals that have both an anaesthetic department and an emergency department (ED). We excluded hospitals whose ED only caters for psychiatric patients. These inclusion and exclusion criteria were selected in order to encompass hospitals that would be expected to manage the airway of a COVID-19 positive patient requiring intubation. Twenty-nine hospitals were invited to be included.

An invitation email was sent to the directors of the anaesthetic departments of each of the 29 selected hospitals. They then nominated the most appropriate person to complete the predesigned survey regarding the airway management of suspected or confirmed COVID-19 patients in their hospital. The nominated person was considered to be the leader or one of the leaders in the hospital, involved in the planning and development of the hospital COVID-19 airway management policies or guidelines. The survey consisted of questions regarding the presence and components of COVID-19 intubation teams, intubation environments, personal protective equipment (PPE), airway management equipment, staff preparation and airway management practices (see supplemental material). These questions were designed to allow comparison across these areas, in order to assess inter-hospital variation in practice. We contacted the nominated person via email. The survey was then completed via their preferred method (a phone call from one of the investigators or self-completion and return by email). Consent was implied by their response to our email and the completion of the survey.

The survey took place during September 2020, coinciding with the declining phase of the second wave of COVID-19 cases in Victoria. There were 723 daily cases at the peak in July 2020. The first wave occurred in March 2020. Most hospitals had developed a policy on airway management of suspected or confirmed COVID-19 patients by the time the study was conducted.

Results from the survey were transferred to a standard spreadsheet (Excel; Microsoft, Redmond, WA, USA), and descriptive statistics were used to present all of the data.

\section{Results}

Of the 29 hospitals contacted, we received responses from 20 hospitals, giving a response rate of 69 per cent. This included 18 public hospitals (12 metropolitan, six regional) and two private hospitals (both metropolitan). All of the surveys were completed by consultant anaesthetists, three of whom were the director of the anaesthetic department. They were all involved in the development of local COVID-19 airway management policies.

Preparation of the intubation team staff for airway management of suspected or confirmed COVID-19 patients is shown in Table 1. Eight hospitals had established dedicated COVID-19 intubation teams, all of which included consultant anaesthetists, during both in-hours and after-hours periods. Of these eight teams, seven included one nurse only. Three included an ICU nurse, three included an anaesthetic nurse, one included an Emergency Department (ED) nurse and one included two nurses from either ICU, anaesthetics or ED. Ninety-five 
Table 1: Preparation of intubation team staff for airway management of suspected or confirmed COVID-19 patients

\begin{tabular}{|l|c|}
\hline Preparation and training & No. of hospitals $(\mathrm{n}=\mathbf{2 0})$ \\
\hline $\begin{array}{l}\text { Intubation staff were fit tested } \\
\text { quantitative:qualitative }\end{array}$ & $7(35 \%)$ \\
\hline Use of cognitive aid for airway management & $18(90 \%)$ \\
\hline Use of simulation training & $17(85 \%)$ \\
\hline $\begin{array}{l}\text { More than 80\% of staff involved in intubation } \\
\text { received formal training in: }\end{array}$ & $19(95 \%)$ \\
- donning/doffing & $19(95 \%)$ \\
- extubation of COVID-19 patients & $16(80 \%)$ \\
\hline
\end{tabular}

percent of hospitals provided formal training for personnel involved in COVID-19 airway management, with $85 \%$ of hospitals using simulation for this purpose (Table 1). Ninety percent of hospitals used a cognitive aid to assist with airway management of these patients.

Most hospitals (18 of 20; $95 \%$ ) provided dedicated COVID-19 intubation rooms, with 75 per cent of hospitals (15 of 20) having negatively pressured rooms for this purpose, frequently in the intensive care unit (ICU) and ED (Table 2). The median number of dedicated intubation rooms per hospital was three (ranging between 1 and 12). Seventyfive percent of hospitals reported the use of a dedicated COVID-19 airway trolley (or other transport means such as a container or bucket).

All of the hospitals had oropharyngeal airways, alternative facemasks/laryngeal masks/ endotracheal tubes and equipment for front-of-neck access either immediately available in the room or closely available outside the room. Ninety-five percent of hospitals (19 of 20) had their videolaryngoscope and bougie inside the room. Only one hospital did not have a hyper-angulated laryngoscope available. However, six of the hospitals (30\%) did not have immediate access to a fibreoptic bronchoscope if required. A variety of protocols were used in different hospitals for both intubation and extubation procedures, as shown in Table 3.

In terms of PPE, all of the hospitals provided gowns, gloves, hats, N95

Table 2: General approach for the airway management of suspected or confirmed COVID-19 patients

\begin{tabular}{|l|c|}
\hline Intubation team and environment & No. of hospitals ( $=20)$ \\
\hline Dedicated intubation team for COVID patients & $8(40 \%)$ \\
\hline Same team during afterhours & $4 / 8(50 \%)$ \\
\hline Dedicated intubation room(s) & $18(90 \%)$ \\
\hline $\begin{array}{l}\text { Dedicated intubation room(s) that are negatively } \\
\text { pressured }\end{array}$ & $15 / 18(83 \%)$ \\
\hline Dedicated intubation trolley & $15(75 \%)$ \\
\hline Videolaryngoscopes available at all times & $20(100 \%)$ \\
\hline Intubation practice & $13(65 \%)$ \\
\hline Limit maximum oxygen flow & $3(15 \%)$ \\
\hline Bag-mask ventilation not allowed & $10(50 \%)$ \\
\hline Protocol for medications to be used & $15(75 \%)$ \\
\hline Mandated clamping of endotracheal tube & \\
\hline
\end{tabular}


Table 3: Details of the protocols used for airway management of suspected or known COVID-19 patients

\begin{tabular}{|c|c|c|}
\hline & \\
\hline & Yes & No \\
\hline Pre-oxygenation protocol & $13(65 \%)$ & $7(35 \%)$ \\
\hline Limit to maximum of $4 \mathrm{~L} / \mathrm{min}$ & 1 & \\
\hline Limit to maximum of $6 \mathrm{~L} / \mathrm{min}$ & 10 & \\
\hline Limit to maximum of $15 \mathrm{~L} / \mathrm{min}$ & 2 & \\
\hline BMV protocol & $14(70 \%)$ & $6(30 \%)$ \\
\hline BMV not allowed & 3 & \\
\hline Allowed if low SpO2 & 11 & \\
\hline - $\mathrm{SpO}_{2}<80 \%$ & 3 & \\
\hline - Anaesthetist's discretion & 7 & \\
\hline - Life threatening situation & 1 & \\
\hline Medication protocol & $10(50 \%)$ & $10(50 \%)$ \\
\hline Induction agent protocolised & 7 & \\
\hline - Ketamine & 4 & \\
\hline - Propofol & 3 & \\
\hline NMB protocolised & 9 & \\
\hline - Rocuronium & 6 & \\
\hline - Suxamethonium & 3 & \\
\hline Opioid protocolised & 5 & \\
\hline - Fentanyl & 3 & \\
\hline - Alfentanil & 2 & \\
\hline Extubation protocol & $9(45 \%)$ & $11(55 \%)$ \\
\hline Plastic sheet & 8 & \\
\hline Deep extubation & 2 & \\
\hline NIV protocol* & $14(78 \%)$ & $4(22 \%)$ \\
\hline Allowed & 8 & \\
\hline Prohibited & 6 & \\
\hline HFNP protocol & $17(85 \%)$ & $3(15 \%)$ \\
\hline Allowed & 13 & \\
\hline Prohibited & 4 & \\
\hline
\end{tabular}

*NIV protocol - two hospitals did not respond $(n=18)$

$\mathrm{BMV}=$ bag-mask ventilation; $\mathrm{SpO2}=$ oxygen saturation with pulse oximetry; $\mathrm{NMB}=$ neuromuscular blockade; NIV = non-invasive ventilation; HFNP = high-flow nasal prongs

\section{Discussion}

All of the 20 responding Victorian hospitals had a policy on airway management of suspected or confirmed COVID-19 patients. However, we found a noticeable amount of variation, not only in the intubation procedure protocols, but also in preparation, training, intubation environment, team structure and PPE use. Multiple factors could have potentially contributed to these variations. They include:

1. lack of evidence to guide a definitive protocol

2. different staff resource capacity

3. different levels of suspected and/ or confirmed COVID-19 cases due to different geographic locations

4. environmental constraint

5. different levels of equipment supply and availability.

Moreover, the lack of an overarching health care structure, which would assist in the integration of resources and sharing of information among different health care networks in victoria, may also have been a potential contributing factor.

It has been suggested that dedicated and experienced COVID-19 intubation teams, such as those using anaesthetists or anaesthetic registrars as lead intubators, may improve patient outcomes and staff safety $^{14}$. This is understandably challenging for some regional hospitals, where the case load is low and resources are limited. Nonetheless, major metropolitan hospitals should consider establishing a COVID-19 intubation team, absent in more than half of the metropolitan hospitals in this study.

Several consensus guidelines specific to airway management of suspected and confirmed COVID-19 positive patients recommend that negative pressure rooms should be 
used where possible during tracheal intubation of these patients, in order to minimise staff exposure to aerosol and droplet particles ${ }^{3,7}$. This is obviously constrained by the hospital environment. The majority of the studied hospitals had dedicated COVID-19 intubation rooms, most of which were negatively pressured. However, we noticed that most COVID-19 intubation rooms that were located in the operating theatre environment were not negatively pressured. The Safe Airway Society consensus statement about principles of airway management and tracheal intubation specific to the COVID-19 adult patient group reports that positive pressure ventilation environments are common in the operating theatre and should be avoided $^{7}$

The use of airborne precautions is recommended in the intubation of high risk (suspected or confirmed COVID-19 positive) patients ${ }^{15,16}$. It is reassuring that all hospitals provided N95 masks, gowns and eye shields for intubation of suspected and confirmed COVID-19 positive patients. Only a minority provided the option of the use of PAPRs or elastomeric respirators. The use of PAPRS or elastomeric respirators is controversial. A recent Cochrane review concludes that the use of a PAPR with coverall may protect against the risk of contamination better than an N95 mask and gown ${ }^{17}$. However, there are concerns regarding their supply and training in donning, doffing and cleaning. Similarly, most hospitals did not provide neck covers for use during intubation of these patients. Interestingly, studies using fluorescent markers as a surrogate measure for contamination have suggested that the neck may be a high risk setting for potential contamination during intubation ${ }^{18}$; however, most guidelines, including the WHO guideline, do not recommend the use of head and neck covers in this setting ${ }^{19}$.

The Australian Guidelines for the Prevention and Control of Infection in Health care state that N95/P2 masks require formal fit testing, in order to identify suitable size and style of mask, and to ensure their correct use ${ }^{20}$. Despite this, most of the hospitals included in this study did not provide N95 mask fit testing, which can be expensive and logistically challenging. It can also consume an extensive number of N95 masks, which may not be ideal during a pandemic ${ }^{21}$. However, this is likely to have improved as the Department of Health and Human Services (DHHS) has recently developed and supported the Respiratory Protection Program at various Victorian health services $^{22}$.

In the management of cardiac arrest of patients suspected or known to be COVID-19 positive, it is reassuring that every hospital provided the equivalent PPE as that worn during airway management of this patient cohort, which is in keeping with recommendations from the $\mathrm{DHHS}^{23}$. Unless there is clear evidence to the contrary, the Australian College of Emergency Medicine (ACEM) recommends that while there are high rates of community transmission of COVID-19 any collapsed or unresponsive patient should be assumed to be high-risk for COVID-19, and that airborne PPE should be $w_{0 r}{ }^{24}$. The significantly higher adaptation of this recommendation for airborne precautions in this setting in metropolitan hospitals, compared to regional hospitals, potentially reflects the higher prevalence of COVID-19 in the metropolitan regions.

One of the potential factors leading to variations in the COVID-19 airway management among different hospitals is the lack of evidence in literature to guide practices. One major uncertainty or controversy is the degree of aerosolisation of COVID-19 during airway manipulation. This may have contributed to the inter-hospital variability seen in the limitation of oxygen flows for preoxygenation, the decision path and trigger for bag-mask ventilation, the protocolisation of drugs used on induction, the mandated clamping of the endotracheal tube for circuit disconnections, extubation practices and the use of high-flow nasal prongs and non-invasive ventilation. This cross-sectional study highlights a large variation in practice among hospitals, despite increasing numbers of publications that are emerging to provide more understanding about aerosol generation during airway management ${ }^{25,26}$.

\section{Limitations}

There are several limitations in this study. It involved only 20 Victorian hospitals, which is a small sample size. However, we included all of the major metropolitan hospitals and many regional hospitals and we believe this is a reasonable representation of Victorian hospitals. There was a selection bias, as we included only the hospitals that responded to our email invitation. Inter-hospital variation in who was nominated to complete the survey and how this decision was made may be present. There is an assumption that individual hospital airway management practices align with their policies, where this may not be the case. Finally, this study is only a snapshot of practices across different Victorian hospitals in September 2020. There are constant changes in COVID-19 management guidelines and practices as new information continues to emerge. Nonetheless, this cross-sectional study highlights a large variation across different hospitals. 


\section{Conclusion}

In conclusion, this study compared how different hospitals in Victoria prepare, coordinate and conduct the airway management of patients with suspected or confirmed COVID-19. We found inter-hospital variability in many areas. This study can be used as a reference and may potentially highlight areas for improvement for some hospitals. Further studies would be beneficial to investigate the reasons behind the variations, and to examine whether there is less variation in other states which have an overarching health care structure. We should also consider the implications of these differences, for example, potential differences in the quality of care of COVID-19 positive patients, potential differences in transmission risk within hospitals and staff wellbeing impacts regarding conflicting information and management of these patients in different locations.

\section{Declaration of conflicting interests}

There are no potential conflicts of interest with respect to this research, authorship, and/or publication of this article.

\section{Funding}

The authors disclose no financial support for this research, authorship, and/or publication of this article.

\section{References}

1. World health Organization (WHO). Timeline: WHO's COVID-19 Response [Internet]. Geneva: WHO; 2020 [cited 2021 Jan 20]. Available from: www.who.int/emergencies/ diseases/novel-coronavirus-2019/ interactive-timeline.

2. World Health Organization (WHO). Emergencies preparedness, response: Disease outbreaks by year 2020 [Internet]. Geneva: WHO; 2020 [cited 2020 Dec 4]. Formerly available from: www.who.int/csr/ don/archive/year/en.
3. Australian Society of Anaesthetists (ASA). Anaesthesia and caring for patients during the COVID-19 outbreak [Internet]. Sydney: ASA; 2020 [cited 2020 Dec 4]. Available from: www.asa.org.au/wordpress/wp-content/ uploads/News/eNews/covid-19/ASA_airway_ management.pdf.

4. Todd B. The toll of COVID-19 on health care workers remains unknown. AJN 2021;121(3):14-15

5. El-Boghdadly K, Wong DJN, Owen R, Neuman MD, Pocock S, Carlisle JB et al. Risks to health care workers following tracheal intubation of patients with COVID-19: A prospective international multicentre cohort study. Anaesthesia 2020;75(11):1437-1447.

6. Stapleton, H. COVID changes to Post Anaesthesia Care Unit nursing practices JPN 2021;34(2):e-19-e-21. DOI: doi. org/10.26550/2209-1092.1113

7. Brewster DJ, Chrimes N, Do TB, Fraser K, Groombridge CJ, Higgs A et al. Consensus statement: Safe Airway Society principles of airway management and tracheal intubation specific to the COVID-19 adult patient group. Med J Aust 2020;212(10):472-481.

8. Zucco L, Levy N, Ketchandji D, Aziz M, Ramachandran SK. An update on the perioperative considerations for COVID-19 severe acute respiratory syndrome coronavirus-2 (SARS-CoV-2). APSF Newsletter 2020;35(2)

9. Australian and New Zealand Intensive Care Society (ANZICS). COVID-19 Guidelines Version 2 [Internet]. Melbourne: ANZICS; 2020 [cited 2021 Feb 14]. Available from: www.anzics.com.au/wp-content/ uploads/2020/04/ANZI_3367_Guidelines_ V2.pdf.

10. Ip VHY, Sondekoppam RV, Özelsel TJP, Tsui BCH. COVID-19 pandemic: International variation of personal protective equipment and infection prevention and control guidelines. Anesth Analg 2020;131(2):e113-e114

11. Grayson L. Politics lies at the heart of the Victorian Health Department's problems [Internet]. Melbourne: The Age; 2020 [7 July 2020; cited 2021 Feb 14]. Available from: www.theage.com.au/national/victoria/ politics-lies-at-the-heart-of-the-victorianhealth-department-s-problems-20200707p559pp.html.

12. Henderson Z. Victoria's health care disaster the result of a privatised system [Internet]. Sydney: Green Left; 2020 [8 September 2020; cited 2021 Feb 14]. Available from: www.greenleft.org.au/content/victoriashealth-care-disaster-result-privatisedsystem.
13. University of Melbourne. Our Affiliates [Internet]. Melbourne: University of Melbourne; 2020 [cited 2020 Jul 7]. Available from: www.healthsciences.unimelb.edu.au/ departments/nursing/engagement/ouraffiliates.

14. Ahmad I, Jeyarajah J, Nair G, Ragbourne SC, Vowles B, Wong DJN et al. A prospective, observational, cohort study of airway management of patients with COVID-19 by specialist tracheal intubation teams. Can J Anaesth 2021; 68(2):196-203.

15. Australian and New Zealand Society of Anaesthetists (ANZCA). ANZCA statement on personal protection equipment (PPE) during the SARS-CoV-2 pandemic: Version 4 [Internet]. Melbourne: ANZCA; 2020 [cited 2021 Feb 14] Available from: www.anzca.edu.au/resources/professionaldocuments/statements/anzca-covid-ppestatement.pdf.

16. Perkins R, Ingebretson E, Holifield L, Bergeron A. CE: A Nurse's Guide to COVID-19. AJN 2021;121(3):28-38.

17. Verbeek JH, Rajamaki B, ljaz S, Sauni R, Toomey E, Blackwood B et al. Personal protective equipment for preventing highly infectious diseases due to exposure to contaminated body fluids in health care staff. Cochrane Database of Systematic Reviews 2020;(5)5

18. Feldman O, Meir M, Shavit D, Idelman R, Shavit I. Exposure to a surrogate measure of contamination from simulated patients by emergency department personnel wearing personal protective equipment. JAMA 2020;323(20):2091-2093.

19. World Health Organization (WHO). Rational use of personal protective equipment for coronavirus disease ("COVID-19)" and considerations during severe shortages: Interim guidance [Internet]. Geneva: WHO; 2020 [6 April 2020; cited 2021 Feb 14]. Available from: apps.who.int/iris/bitstream/ handle/10665/331695/WHO-2019-nCovIPC_PPE_use-2020.3-eng.pdf.

20. National Health and Medical Research Council (NHMRC). Australian Guidelines for the prevention and control of infection in healthcare. Canberra: NHMRC;2019.

21. Regli A, von Ungern-Sternberg BS. Fit testing of $\mathrm{N} 95$ or $\mathrm{P} 2$ masks to protect health care workers. Med J Aust 2020;213(7):293-5.e1.

22. Department of Health and Human Services (DHHS). Protecting our healthcare workers [Internet]. Melbourne: DHHS;2020 [cited 2021 Jan 20]. Available from: www.dhhs.vic.gov.au/sites/default/files/ documents/202010/Protecting-ourhealthcare-workers-action-plan.pdf. 
23. Department of Health and Human Services (DHHS). Managing adult patient rapid response calls for known or suspected COVID-19 patients [Internet]. Melbourne: DHHS; 2020 [cited 2021 Jan 20]. Available from: www.dhhs.vic.gov.au/factsheet-rapidresponse-call-management-known-orsuspected-covid-19-patients.

24. Australasian College of Emergency Medicine (ACEM). Adult cardiac arrest management [Internet]. Melbourne: ACEM; 2020 [cited 2020 Nov 10]. Available from: acem.org.au/Content-Sources/AdvancingEmergency-Medicine/COVID-19/Resources/ Clinical-Guidelines/Adult-Cardiac-ArrestManagement.

25. Gaeckle NT, Lee J, Park Y, Kreykes G, Evans $M D$, Hogan CJ. Aerosol generation from the respiratory tract with various modes of oxygen delivery. Am J Respir 2020;202(8):11151124.

26. Kangas-Dick AW, Swearingen $B$, Wan E, Chawla K, Wiesel O. Safe extubation during the COVID-19 pandemic. Respir Med 2020;170:106038. 


\section{Variations in COVID-19 airway management and preparedness among Victorian hospitals}

\section{Supplementary material}

An audit on COVID-19 airway management across Victorian Hospitals

Project lead person: Dr Kaylee Jordan

Other key personnel: Prof. Reny Segal, Dr Irene Ng, Dr Keat Lee, Dr Roni Krieser, Dr Paul Mezzavia, Ms Teresa Sindoni, Ms Yinwei Chen

\section{General Information}

Name of hospital:

Major metropolitan

$\square$ Public - metropolitan

$\square$ Private - metropolitan

\section{Teams}

2.1 Do you have a dedicated COVID-19 intubation team at your hospital?

$\square$ Yes $\square$ No

2.2 If so, who makes up the team? (how many)

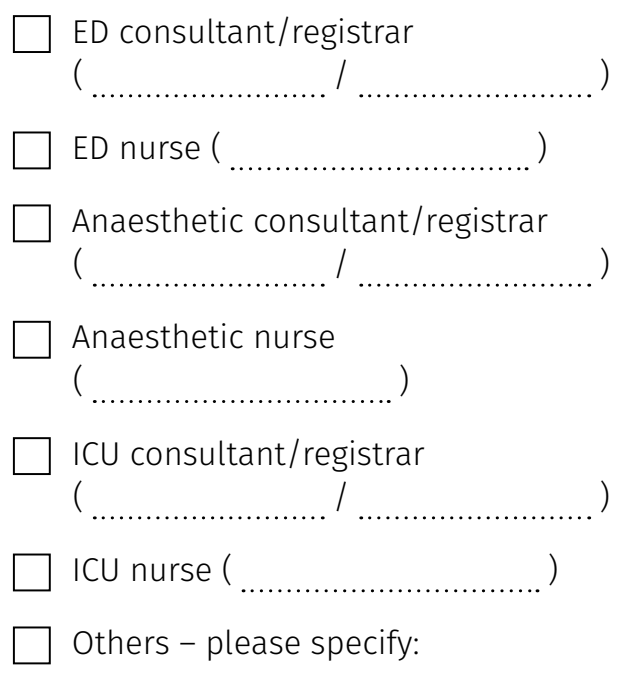

2.3 Are all members of this team included in the after-hours COVID-19 intubation team?

Yes

$\square$ No (specify who is not in this team after hours)
2.4 Who makes up the Code Blue team for suspected/known COVID-19 patients?

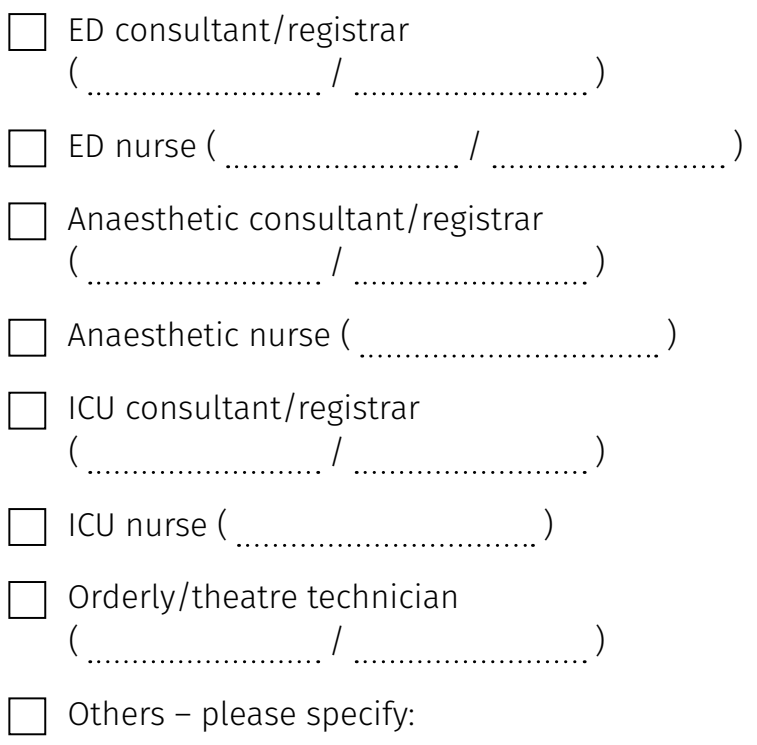

\section{Environment}

3.1 Do you have dedicated Covid-19 intubation rooms in the hospital?

$\square$ Yes (How many and where:

$\square$ No

3.2 If so, are these rooms negatively pressured?

$\square$ Yes $\square$ No

Others - please specify: 
4. PPE

4.1 When intubating a suspected/known Covid-19 patient, which of the following PPE equipment is required to be worn at your hospital? (tick all that apply)

$\square$ Gown (long sleeved/short sleeved)

$\square$ Hat

$\square$ Neck cover

$\square$ Goggles

$\square$ Eye shield

N95 mask

$\square$ P2 mask

Powered Air Purifying Respirator (PAPR)

$\square$ Shoe covers

$\square$ Others - please specify:

4.2 Does your hospital/department provide N95 mask fit testing for staff who are involved in intubation of suspected/known COVID-19 positive patients?

$\square$ Yes (quantitative/qualitative)

$\square$ No

4.3 Are the same protective equipment worn for attendance at cardiac arrests of patients suspected/known COVID-19 positive?

$\square$ Yes

$\square$ No (specify which of the items ticked above are not worn

4.4 Are the same protective equipment worn for attendance at cardiac arrests of patients not suspected/known to be COVID-19 positive?

$\square$ Yes

$\square$ No (specify which of the items ticked above are not worn

\section{Equipment}

5.1 Is there a dedicated COVID-19 airway trolley in your hospital?

$\square$ Yes $\square$ No

5.2 Regarding the following equipment, please select whether each are available during a suspected/known Covid19 intubation, in your department either:
a) in the room
b) outside the room or
c) not available.

\begin{tabular}{|l|l|l|l|}
\hline Equipment & $\begin{array}{c}\text { In the } \\
\text { room }\end{array}$ & $\begin{array}{c}\text { Outside } \\
\text { the room }\end{array}$ & $\begin{array}{c}\text { Not } \\
\text { available }\end{array}$ \\
\hline Alternative facemasks & & & \\
\hline Guedels & & & \\
\hline Videolaryngoscope & & & \\
\hline $\begin{array}{l}\text { Hyperangulated } \\
\text { laryngoscope }\end{array}$ & & & \\
\hline Alternative ETTs & & & \\
\hline Bougie & & & \\
\hline Stylet & & & \\
\hline Alternative LMAs & & & \\
\hline $\begin{array}{l}\text { CICO kit: Needle } \\
\text { cricothyroidotomy } \\
\text { Scalpel/bougie Melker }\end{array}$ & & & \\
\hline Fibreoptic bronchscope & & & \\
\hline
\end{tabular}

5.3 Does your hospital/department use cognitive aid for airway management of suspected/known COVID-19 patients?

$\square$ Yes $\square$ No 


\section{Preparation}

6.1 Have the majority ( $>80 \%$ ) of your team received formal training in (tick those that apply)

\section{$\square$ Donning/doffing}

$\square$ Intubation of a suspected/known COVID-19 positive patient

Extubation of a suspected/known COVID-19 positive patient

6.2 Does your hospital/department use simulation training for management of suspected/known COVID-19 positive patients?
$\square$ Yes
No

\section{Practice}

7.1 When inducing someone with suspected or known COVID-19 in your department, do you have a protocol for maximum oxygen flows to be used?

$\square$ Yes (please specify )

$\square$ No

7.2 When inducing someone with suspected or known COVID-19 in your department, do you have a protocol for medications to be used?

$\square$ Yes (please specify )

$\square$ No

7.3 When inducing someone with suspected or known COVID-19 in your department, do you have a protocol specifying whether/ when you should bag mask ventilate (BMV)?

No protocol

$\square$ Protocol - routinely BMV

$\square$ Protocol - never BMV

$\square$ Protocol - BMV only if oxygen saturations fall below a trigger

(specify
7.4 When intubating a patient with known or suspected COVID-19 in your department, is a video laryngoscopy available for all intubations?

$\square$ Available at all times

$\square$ Limited availability

$\square$ No availability

7.5 When swapping between equipment for ventilation (e.g. between ventilators or self-inflating bag to ventilator), does your hospital/department mandate clamping of the ETT?

$\square$ Yes $\square$ No

7.6 When extubating a patient with suspected or known COVID-19 in your department, does your hospital protocol recommend any of the following precautions?
Plastic bag
$\square$ Plastic box
$\square$ Deep extubation
$\square$ Other (please specify

When managing COVID positive patients in your department, does your hospital specify whether either of the following may be used if required?

a) NIV

$\square$ No protocol

$\square$ Prohibited

$\square$ Allowed

b) HFNP

$\square$ No protocol

Prohibited

Allowed 